\title{
SEAMLESS NAVIGATION USING VARIOUS SENSORS: AN OVERVIEW OF THE SEAMLESS NAVIGATION CAMPAIGN
}

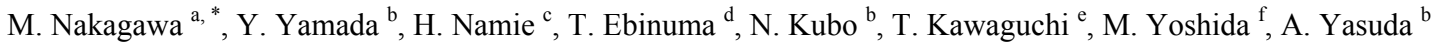 \\ ${ }^{a}$ Shibaura Institute of Technology, 3-7-5, Toyosu, Koto-ku, Tokyo, 135-8548, Japan - mnaka@shibaura-it.ac.jp \\ ${ }^{\mathrm{b}}$ Tokyo University of Marine Science and Technology - (m105004, nkubo, yasuda)@kaiyodai.ac.jp \\ ${ }^{\mathrm{c}}$ The National Defense Academy of Japan - nami@nda.ac.jp \\ ${ }^{\mathrm{d}}$ University of Tokyo - ebinuma@nsat.t.u-tokyo.ac.jp \\ ${ }^{\mathrm{e}}$ Hitachi Industrial Equipment Systems Co.,Ltd - takamasa.kawaguchi.cj@hitachi.com \\ ${ }^{\mathrm{f}}$ Salesian Polytechnic - yoshida@salesio-sp.ac.jp
}

Commission IV, WG IV/1

KEY WORDS: Seamless positioning, Global Navigation Satellite System, Indoor positioning

\begin{abstract}
:
Seamless positioning techniques in indoor and outdoor environments are necessary for obtaining sensor locations. However, no definitive indoor-outdoor navigation system simultaneously provides high accuracy, high availability and low installation cost. Furthermore, crowded indoor-outdoor navigation systems consisting of multiple techniques will destructively interfere with each other, but an exclusive navigation environment will have difficulty providing stable location services for users. This anticipated issue needs to be investigated with experimental data and simulation results. However, experiments that are deliberately overcrowded with disparate location systems are rare. Therefore, the initial focus in our research was the construction of a test environment for indoor-outdoor seamless navigation experiments. Based on "Standards and Recommended Practices" (SARPs), we focused on accuracy, availability, continuity and integrity to verify the effects of seamless navigation under a combination of as many disparate systems and sensors as possible. We then conducted data acquisition and data analysis in seamless navigation through four integrated experiments. Based on the results of our experiments, we summarize some observations about seamless navigation using multiple navigation systems, and offer examples of the representative issues in our research. We also suggest some directions in indoor-outdoor navigation environment construction for seamless positioning using disparate systems and sensors.
\end{abstract}

\section{INTRODUCTION}

Currently, in many fields such as navigation, disaster relief and construction automation, seamless positioning techniques in indoor and outdoor environments are necessary for obtaining sensor locations. However, no definitive indoor-outdoor navigation system simultaneously provides high accuracy, high availability and low installation cost. The number of sensors installed in mobile devices has increased over the years, and in particular, many mobile devices equipped with multiple location sensors will continue to be developed in the future. Therefore, for commercial uses, multiple navigation systems will be installed in indoor-outdoor environments in urban areas. However, crowded indoor-outdoor navigation systems consisting of multiple techniques will destructively interfere with each other, but an exclusive navigation environment will have difficulty providing stable location services for users. This anticipated issue needs to be investigated with experimental data and simulation results.

However, experiments that are deliberately overcrowded with disparate location systems are rare. Therefore, the initial focus in our research was the construction of a test environment for indoor-outdoor seamless navigation experiments. Based on "Standards and Recommended Practices" (SARPs), we focused on accuracy, availability, continuity and integrity in order to test seamless navigation under a combination of as many different systems and sensors as possible.

\section{EXPERIMENTS}

In this environment, we placed lighting tags, an Indoor Messaging System (IMES) [1], Radio Frequency Identification (RFID) tags, a Quasi-Zenith Satellite Systems (QZSS) [2] receiver, a high-sensitivity GPS/GLONASS receiver, an Attitude and Heading Reference System, a time-of-flight infrared camera, a laser scanner and an omnidirectional camera, as shown in Table 1. We integrated these sensors for use as an indoor-outdoor navigation system, an indoor-limited navigation system and a pedestrian tracking system.

Table 1. Sensors used in the test environment

\begin{tabular}{|l|l|}
\hline Sensor type & Sensor name \\
\hline Quasi-Zenith Satellite (QZS) receiver & DELTA receiver + GrAnt-3G antenna (JAVAD) \\
\hline High-sensitivity GPS/GLONASS receiver & EVK-5H receiver (u-blox) \\
\hline Indoor Messaging System (IMES) & $\begin{array}{l}\text { SmartModule-IMES Transmitter (HITACHI), } \\
\text { EVK-5H receiver(u-blox) }\end{array}$ \\
\hline Lighting tags & Lighting tags (NEC) \\
\hline Radio Frequency Identification (RFID) tags & MOD_RFID125 (OLIMEX) \\
\hline Attitude and Heading Reference System (AHRS) & MTi/MTi-G (Xsens) \\
\hline Omnidirectional camera & Ladybug2 (Point Grey) \\
\hline Laser scanner & LMS100 (SICK) \\
\hline Time-of-flight infrared camera & SR8000 (MESA Imaging) \\
\hline
\end{tabular}


We conducted experiments using listed sensors through four integrated experiments and then analyzed the data. The first experiment involved a coarse-resolution indoor navigation using position data taken at 22 points to investigate availability and continuity in an indoor navigation environment. The second experiment involved a fine-resolution indoor navigation using position data taken at 254 points with electric field maps generated from each sensor to investigate accuracy and continuity in an indoor navigation environment. The third experiment integrated navigation of both indoor and outdoor environments to investigate availability and continuity in an indoor-outdoor navigation environment. The fourth experiment involved outdoor navigation using multiple satellite systems to investigate accuracy, availability and integrity.

\subsection{Integrated sensor system}

The sensors listed in Table 1 were integrated to test seamless navigation. We prepared three integrations in our experiments, as follows.

\section{Indoor-outdoor navigation system}

Signals from satellites GPS, GLONASS, QZSS and IMES were received simultaneously with a DELTA receiver. These signals were then synchronized with GPS time. The receiver is packed in a backpack with an antenna directed vertically, as shown in Figure 1. Position estimation was conducted in offline processing.

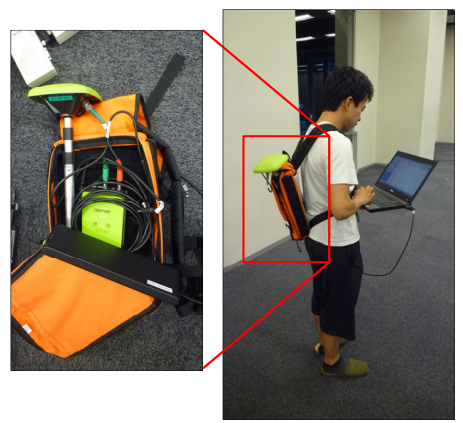

Figure 1. Position data acquisition in an indoor-outdoor environment

\section{Indoor navigation system}

A lighting tag receiver, an IMES receiver and an RFID receiver were integrated as an indoor navigation system. These receivers were connected to a mobile PC and were synchronized with the PC time. Two patterns were tested with this system, as shown in Figure 2.

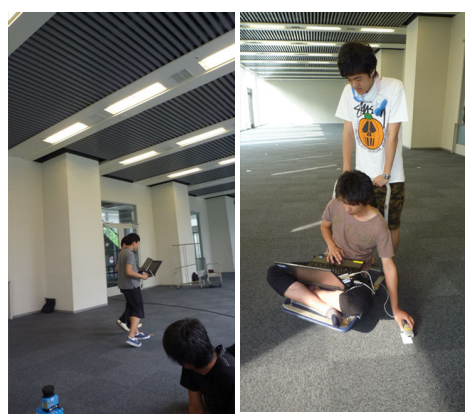

Figure 2. Position data acquisition in an indoor environment
To produce the first pattern, the experimenter walked while holding the mobile PC to simulate navigation for pedestrians. This pattern was focused on the simultaneous use of lighting tags and IMES. Another pattern involved smooth movement by a truck to simulate navigation for autonomous robots. This pattern was focused on the simultaneous use of lighting tags, IMES and RFID tags.

\section{Pedestrian tracking sensor}

An omnidirectional camera and laser scanner were combined to track pedestrians, as shown in Figure 3. The omnidirectional camera captured a panorama movie. The panorama movie was mainly used to synchronize all position sensor data using pedestrian behavior in manual offline processing. The laser scanner was set at a point $30 \mathrm{~cm}$ above the floor. Pedestrian positions were extracted from the temporal laser scanner data using the scene-subtraction methodology.

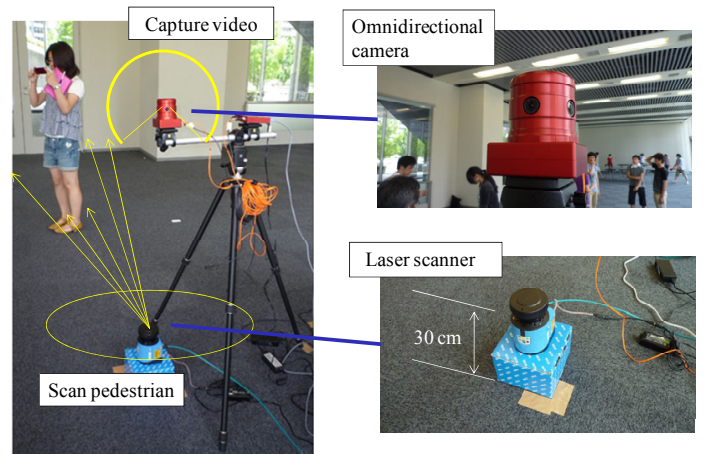

Figure 3. Position data acquisition and pedestrian tracking with an omnidirectional camera and laser scanner

\subsection{Construction of test environment for indoor-outdoor seamless navigation experiments}

For the outdoor experiment, we selected an area around our campus, as shown in Figure 4. This area includes parks, highrise buildings, low-rise buildings, stations and wide and narrow roads. For the indoor experiment, we selected a large room in our campus with an outdoor opening, as shown in Figure 5.

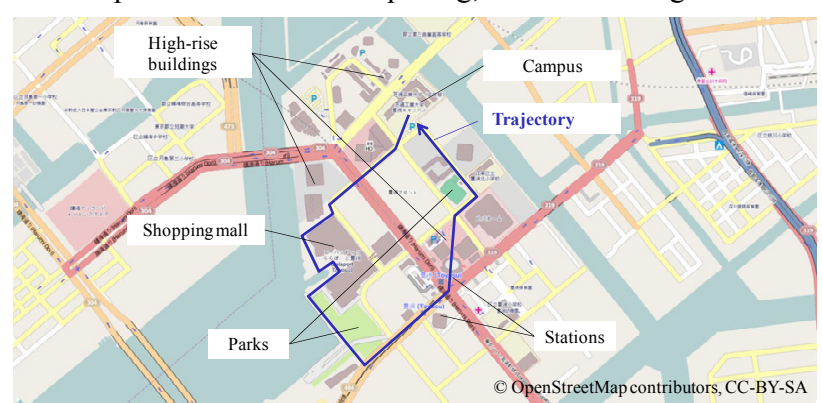

Figure 4. Study area (outdoor)

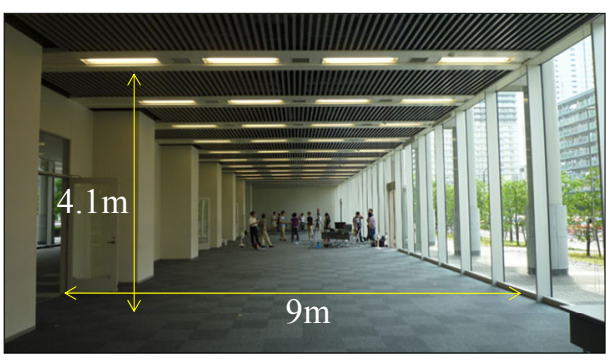

Figure 5. Study area (indoor) 


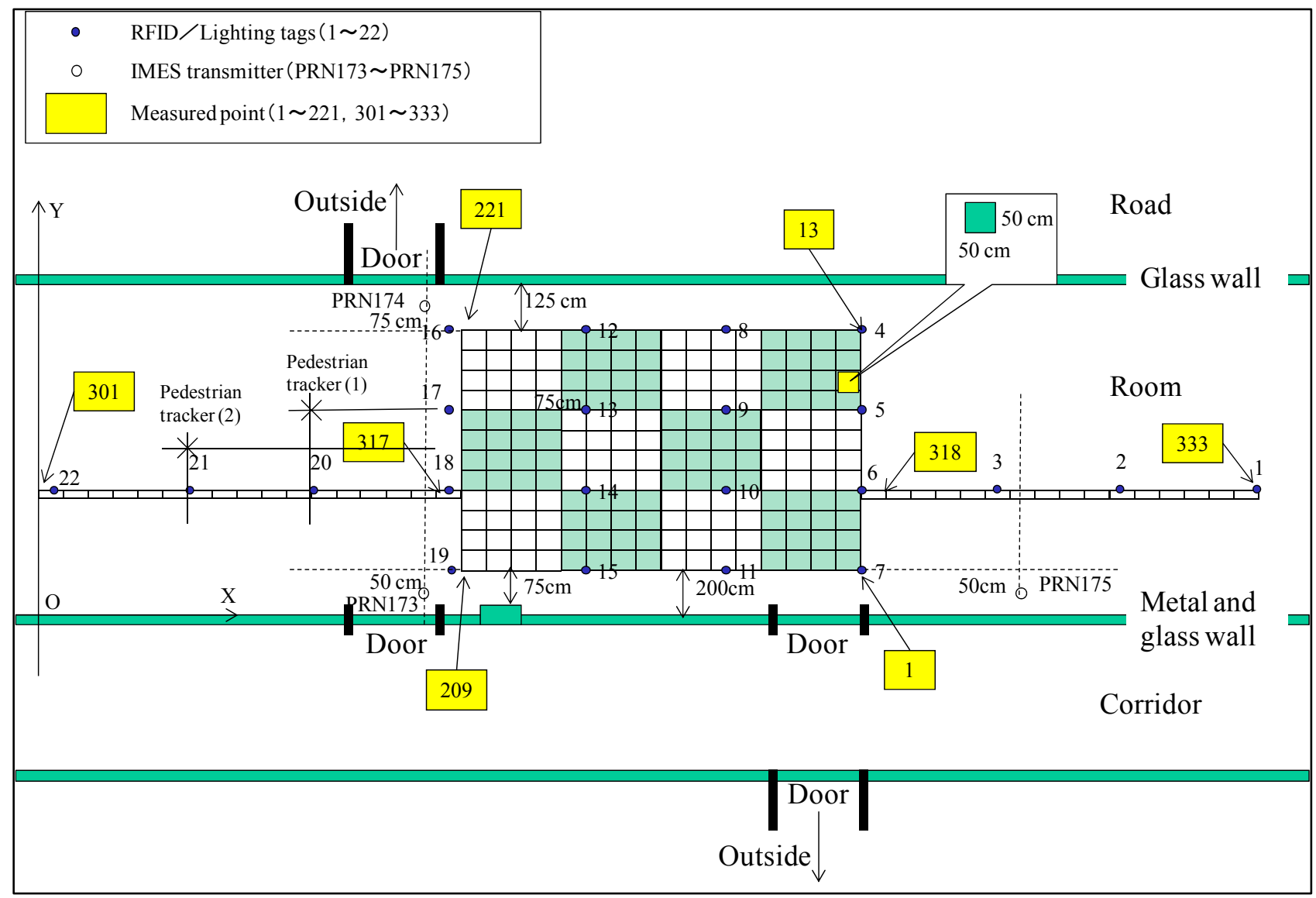

Figure 6. Sensor distribution map for the indoor study area

In the indoor experiment, each sensor was placed in the indoor study area according to our sensor distribution map, shown in Figure 6. Each lighting tag was attached at the center of an inverter light, and was supplied electric power from the inverter, as shown in Figure 7. Each RFID tag was arranged on the floor directly under each lighting tag, as shown in Figure 8. These tags were arranged at 22 points to track pedestrian behavior including walking in a straight line and meandering.

Three IMES transmitters with dipole antennae were installed on the ceiling at corners of our test field, as shown in Figure 9. Each IMES transmitter was assigned a Pseudo Random Noise code (173, 174 or 175), and position data were estimated from short identification numbers received from the transmitters.

Generally, position data are directly taken from the recorded position data within the tags. However, in this experiment, position data were converted from the identification numbers of the tags.

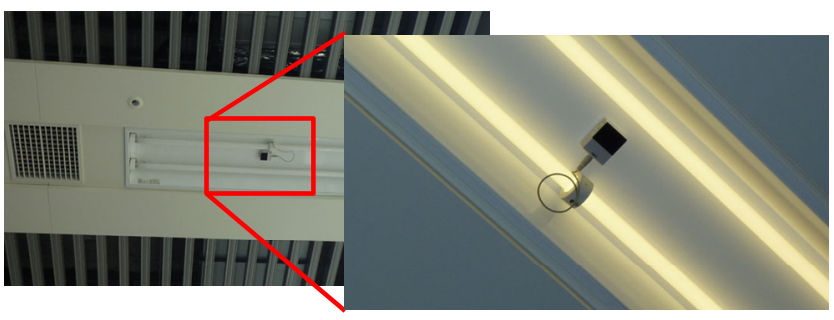

Figure 7. Lighting tags installed on the ceiling

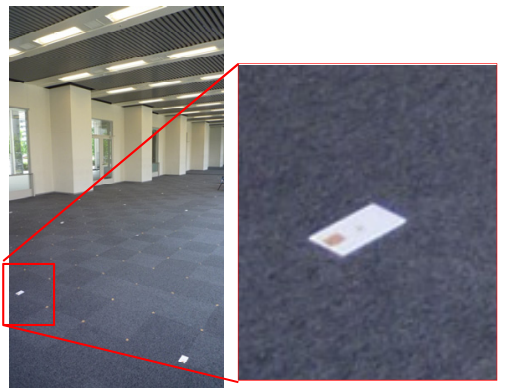

Figure 8. RFID tags installed on the floor

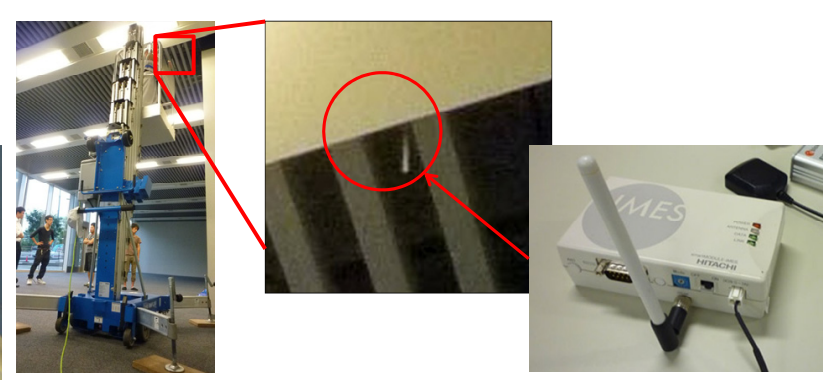

Figure 9. IMES transmitter installed on the ceiling 


\subsection{Results}

Partial experimental results are as follows. Figure 10 presents one of the results from our outdoor experiment for evaluation of the availability when GNSS receivers including GPS, GLONASS and QZSS are integrated in dense urban areas. Figure 11 shows a result from pedestrian tracking taken from laser scanner data in our indoor experiment. This result was used as a reference value for evaluating the performance of positioning sensors. The accuracy, integrity and continuity of each sensor in our experimental environment are summarized in Table 2 (although we recognize that the performance of sensor system depends on an environment).

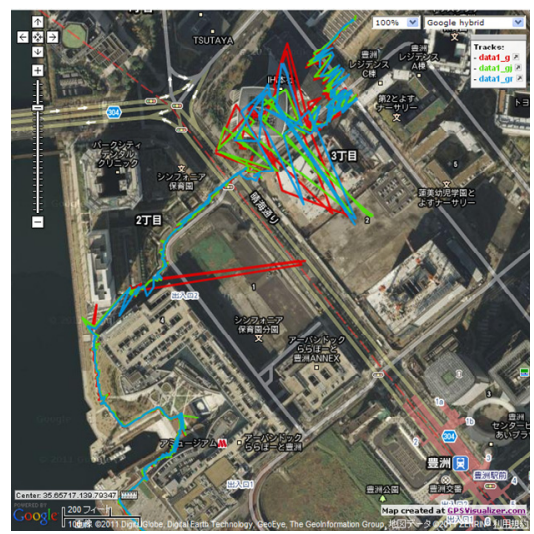

Figure 10. A result from the our outdoor experiment

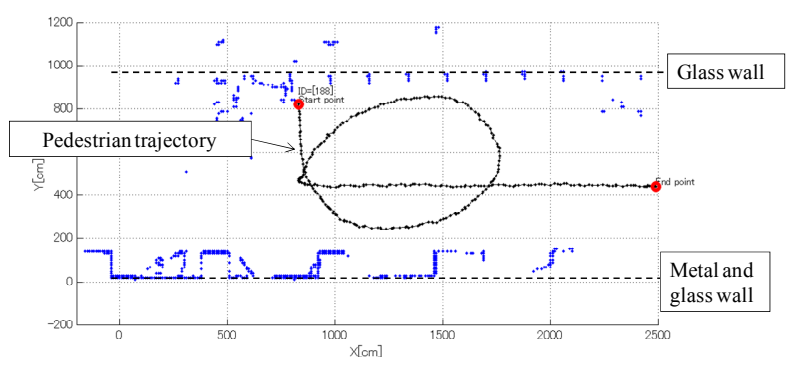

Figure 11. Result from pedestrian tracking using laser scanner data

Table 2. Accuracy, integrity and continuity of each sensor in the experimental environment

\begin{tabular}{|c|c|c|c|c|}
\hline \multicolumn{2}{|c|}{} & IMES & Light tag & RFID tag \\
\hline $\begin{array}{c}\text { Accuracy } \\
\text { (Service area) }\end{array}$ & & $10 \mathrm{~m}$ & $1.5 \mathrm{~m}$ & $0.1 \mathrm{~m}$ \\
\hline \multirow{2}{*}{ Continuity } & Static & $74.67 \%$ & $26.38 \%$ & - \\
\cline { 2 - 5 } & Move & $83.96 \%$ & $97.70 \%$ & - \\
\hline \multirow{2}{*}{ Integrity } & Static & $94.49 \%$ & $57.30 \%$ & $100.00 \%$ \\
\cline { 2 - 5 } & Move & $54.15 \%$ & $51.46 \%$ & $79.75 \%$ \\
\hline
\end{tabular}

\section{DISCUSSION}

The first experiment involved a coarse-resolution indoor navigation using position data taken at 22 points to investigate availability and continuity in an indoor navigation environment. The second experiment involved a fine-resolution indoor navigation using position data taken at 254 points with electric field maps generated from each sensor to investigate accuracy and continuity in an indoor navigation environment. The third experiment integrated navigation of both indoor and outdoor environments to investigate availability and continuity in an indoor-outdoor navigation environment. The fourth experiment involved outdoor navigation using multiple satellite systems to investigate accuracy, availability and integrity.

Based on the results from our experiments, we can make some observations about seamless navigation using multiple navigation systems, focusing on the following representative issues from our research. The first issue is the improvement of availability using multiple navigation systems. We have clarified that outdoor positioning of multiple navigation satellites has the potential to improve availability in an opensky environment. Indeed, compared with the use of GPS only, the use of multiple navigation satellites improved the position fix rate by $20 \%$. On the other hand, we also clarified that in a dense urban environment, the integrity of navigation decreases from $100 \%$ to approximately $50 \%$ because of multipath interference. In addition, we clarified in our experiment that the position fix rate decreases when the number of navigation satellites increases.

The second issue is interference between indoor navigation systems. We found that, because of interference, fewer satellite signals are received by high-sensitivity GPS receivers when an IMES transmitter is located within a $3 \mathrm{~m}$ radius of the receiver. Therefore, we conclude that the design of IMES transmitter arrangements should accommodate this restriction in indooroutdoor border areas for seamless navigation using GPS and IMES.

The third issue is interference between the indoor navigation and 3D measurement systems. When the orientation of time-offlight infrared camera is within $40^{\circ}$ of the line of sight between a lighting tag and a lighting tag receiver, infrared interference renders the lighting tag system unavailable. Therefore, for human sensing and autonomous robot navigation, we must focus on the directional properties of sensors.

\section{SUMMARY}

The initial focus in our research was the construction of a test environment for indoor-outdoor seamless navigation experiments. Based on SARPs, we focused on accuracy, availability, continuity and integrity to verify the effects of seamless navigation under a combination of as many systems and sensors as possible. We then conducted data acquisition and data analysis in seamless navigation through four integrated experiments. Based on the results of our experiments, we summarized some observations about seamless navigation using multiple navigation systems.

\section{References}

[1] Dinesh Manandhar, Seiya Kawaguchi, Masayuki Uchida, Makoto Ishii, Hideyuki Torimoto. 2008. IMES for Mobile Users Social Implementation and Experiments based on Existing Cellular Phones for Seamless Positioning. In: Proceedings of International Symposium 2008 on GPS-GNSS.

[2] S. Kogure, M. Sawabe, and M. Kishimoto. 2006. Status of QZSS Navigation System in Japan. In: Proceedings of ION GNSS 2006, pp. 2092-2102.

\section{Acknowledgements}

This work forms part of a series of experiments conducted by a committee in the Institute of Electrical Engineers of Japan (IEEJ). This work is partly supported by the Strategic Information and Communications R\&D Promotion Programme (SCOPE) of the Ministry of Internal Affairs and Communications, Japan. 